\title{
The dog particle
}

\author{
Gregory A Petsko*
}

\begin{abstract}
A large lecture hall in the main building at CERN, The Canine Experimental Research Network headquarters, somewhere in Europe. The hall is packed with science reporters from many nations. An expectant buzz is heard throughout the room. At the front of the hall is a stage with a podium. TV cameras and microphones are clustered around the front of the stage, aimed at the podium. A door to the right of the stage opens, and a large chocolate Labrador retriever walks across to the podium. A small, wheat-colored spaniel-poodle mixed breed trots after him and stands off to the side. The Labrador begins (Figure 1).
\end{abstract}

Mink: Good morning, I'm Dr Mink, the head of one of the two experimental consortia here. I'll be making the first presentation. It's $\mathrm{OK}$ to ask questions during my remarks, if there's something you don't understand. (Clears throat.) As you know, the Standard Poodle Model of the Universe is perhaps the greatest intellectual achievement of the branch of science called muttaphysics. But you also know that the Standard Poodle Model has been unable to explain one of the deepest mysteries of the cosmos, namely, why a dog's nose is always cold. It doesn't matter if it's cold outside or hot outside; the nose is always cold. It has been appreciated, for many years, that this fact violates the Second Law of Thermodynamics.

Clifford (suddenly singing):

Heat won't pass from a cooler to a hotter;

You can try it if you like, but you far better notter,

'Cause the cold in the cooler will get hotter as a ruler,

'Cause the hotter body's heat will pass to the cooler...

Mink (sharply): Stop that! (To the audience) Excuse me, please. My colleague was just channeling Flanders and Swann. Where was I? Oh, yes. The great mystery of how a dog's nose can stay cold even when it's very hot outside. With the recent discovery of Bark Matter, this is clearly the fundamental problem remaining, and therefore is occupying the attention of muttaphysicists worldwide. You will recall that, in 1974, Hermann Briggs

*Correspondence: petsko@brandeis.edu

Rosenstiel Basic Medical Sciences Research Center, Brandeis University, Waltham, MA 02454-9110, USA

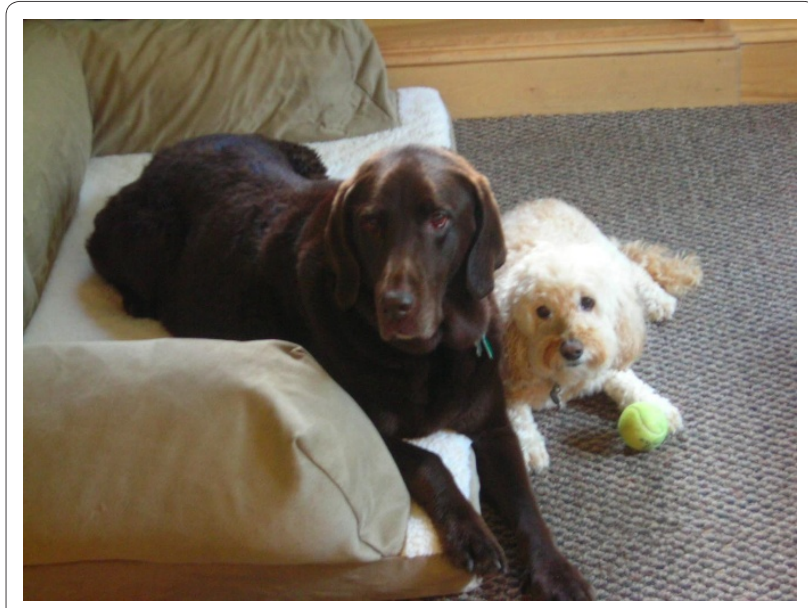

Figure 1. Mink and Clifford proudly show off the effects of the Briggs Noson.

proposed a solution to the problem, by hypothesizing the existence of a particle that could carry heat away from the tip of the nose. The one you journalists sometimes call 'the dog particle.'

Reporter from Nature magazine: You mean...

Mink: That's right: the Briggs Noson. We've called this meeting because we have a monumental discovery to announce with respect to this particle. (An excited murmur races through the room.) You will recall that, in his initial theory, Briggs proposed that the Briggs Noson needed to have a mass somewhere between zero and a gazillion electron volts. Since then, almost 40 years of astronomically expensive high-energy muttaphysics experiments have narrowed that range down to between 1 electron volt and a gazillion electron volts. Now let me show you the new data, about which we are so excited. They come from two completely different, independent experiments. I'll present the results of the first one, and my colleague - (glances at Clifford, who has started to wander off aimlessly) Sit! Stay! - Sorry, my colleague, Dr Clifford, will present the others. You will be amazed at the agreement. (He brings up the first slide on a large screen. It shows a large black dog and smaller white rabbit facing each other in a tunnel.) These data come from the LHC, the Labrador Hare Collider. In this experiment, we smash the cold nose of a Labrador into the warm nose of 
a rabbit and look for evidence of a particle that would transfer heat away from the dog's nose to that of the bunny. (The slide changes to a movie, in which the dog and the rabbit repeatedly butt noses against each other.) After years of data collection at a cost of bazillions of euros, we produced the data shown on the next slide. (The movie is replaced by a slide showing a graph with an enormous amount of noise and two tiny blips a fraction above the noise.) These observations allow us to make a definitive statement about the existence of the Briggs Noson for the first time. But before we do that, my colleague will show the results of the other experiment.

Clifford (stepping to the podium): I'm going to show you the results of the completely independent and totally different experiments from the HLC, the Hound Lepus Collider. The next slide shows the experimental configuration. (Slide changes to show what looks like the same black Labrador and the same white rabbit facing each other in the same tunnel.)

Reporter from Science magazine: That looks like exactly the same experiment as the first one.

Clifford (indignantly): Well, it's not! The LHC experiment is carried out on the third floor of CERN, but my HLC experiment is carried out on the second floor. So you see, the conditions are completely different.

Reporter from Physics Yesterday: And just how much did this experiment cost?

Clifford: At least two bazillion euros. But it was worth it: in the movie that's coming up on the screen now, you will see the actual historic data as they were being collected.

(The screen changes to a film showing, through a transparent window in the tunnel, the dog and the rabbit butting heads. Outside the window, Clifford, Mink, and a number of other scientists are gazing with rapt attention at a huge cathode ray detector that must have cost squintillions of euros. The detector is mostly completely blank. Suddenly a blip occurs.)

Clifford: There! Did you see it? (Another blip appears.) See - there's another one. (This continues for a time.)

Reporter for the News of the World (suddenly): Say, aren't those blips appearing every time the chocolate Lab scratches himself? Could he have been jostling the detector accidentally?

(Mink and Clifford stare at the film for a few moments.)

Mink (hastily changing to the next slide): Er, possibly, but it doesn't change our revolutionary conclusion (the screen shows another set of data, as noisy as the first, with three blips a fraction above the sea of noise).

Reporter for USA Today: You mean, you have proof that the Briggs Noson exists?
Mink: We can't conclude that, no.

Reporter for Oggi: You mean, you have proof the Briggs Noson doesn't exist?

Clifford: We can't conclude that either, no.

Reporter for Der Stern: Well, what exactly can you conclude?

Mink: We can conclude that, if the Briggs Noson does exist, it has a mass between 2 electron volts and a gazillion electron volts. We have been able to narrow down the possible mass range considerably.

Reporter from Japan Times: You at CERN wouldn't be approaching a decision on another round of funding by any chance, would you?

Clifford: Possibly.

Reporter from EMBO Journal (who doesn't really belong here but, like the others, wasn't about to turn down a free trip to Geneva): So this was all just a publicity stunt to get us to write stories that would convince the politicians who pay for this stuff that it's worth pouring more quadrillions of euros into a bunch of experiments that haven't found anything definitive yet and possibly never will! Sheesh - what a letdown! I mean, what if the people who sequenced the human genome had announced that they had completed the sequence but had no idea how many genes there were?

Clifford: Isn't that exactly what they did announce?

Mink: And as for this being all about the publicity and funding, isn't that what most Big Science projects do? They release enough periodic announcements of 'important discoveries' that aren't really all that important when you look at them carefully, and that by coincidence tend to come out more frequently when their funding is up for renewal. Look at the structural genomics projects, and the genome wide association studies, and the cancer genome program, and the -

Reporter from The Australian: But that doesn't mean the muttaphysicists should keep doing it! You Briggs Noson hunters fooled us once before, you know.

Mink: You came this time, didn't you?

(With groans of disgust, the reporters file out of the lecture hall. Mink and Clifford are left alone on the stage.)

Clifford (to Mink): Maybe we finally overdid the hype before this event this time. What if they never come back?

Mink (wisely): Oh, they'll be back. They always come back. After all, they have a nose for news.

Published: 30 January 2012

doi:10.1186/gb-2012-13-1-142

Cite this article as: Petsko GA: The dog particle. Genome Biology 2012, 13:142. 